\title{
Epidemiology of Suspected Pesticide Poisoning in Livestock
}

\author{
Judy Akkina* and Leah Estberg \\ United States Department of Agriculture (USDA), Animal and Plant Health Inspection Service (APHIS), Veterinary Services, \\ Fort Collins, CO, USA
}

\section{Objective}

This study characterizes the epidemiology of suspected pesticide poisoning in livestock in the United States (U.S.) and Canada using data from calls to the American Society for the Prevention of Cruelty to Animals (ASPCA) Animal Poison Control Center (APCC).

\section{Introduction}

Pesticides are used in agriculture and in the home to control pests such as insects, weeds, fungi and rodents. Pesticide poisoning in animals is usually due to misuse or accidental exposure ${ }^{1}$. Information on poisonings in livestock in North America is largely lacking ${ }^{2}$. Examples of hotlines in the U.S. for animal poisoning consultations include the APCC ( $\$ 65.00$ fee) and the Pet Poison Helpline (PPH) ( $\$ 59.00$ fee). The APCC fields calls 24 hours/day, 7 days/week about animal poisonings from the U.S., its territories and Canada. Using data from almost 4 years of APCC calls we describe the occurrence, category and class of pesticides involved, and outcomes of suspected pesticide exposures in livestock. This information is useful to raise awareness, encourage the proper use of pesticides and identify specific pesticides with negative impact on livestock health.

\section{Methods}

APHIS contracts with the APCC to receive de-identified data weekly on livestock calls for the purpose of conducting surveillance. This retrospective study used data from all calls concerning bovine, camelid, caprine, equine, ovine, porcine and poultry species from $10 / 1 / 2013$ to $9 / 2 / 2017$, where the caller reported suspected pesticide exposure. There were 1,025 calls regarding 3,028 animals meeting this criteria, representing $52 \%$ of all livestock calls with any type of toxic exposure. Caller type was $80 \%$ animal owners, $10 \%$ veterinarian or veterinary staff, and $10 \%$ other types. Most callers (92\%) provided their zip code, with $96 \%$ of calls from the U.S. and $4 \%$ from Canada. Variables used for descriptive analysis were: species; APCC staff assessment that illness was due to pesticide exposure; severity of illness; clinical signs; first, second and third ingredients of the pesticide, and pesticide ingredient class (e.g. pyrethrin). Pesticides were grouped based on the first active ingredient into fungicide, herbicide, insecticide, and rodenticide categories.

\section{Results}

The proportion of calls by species was equine $(33 \%)$, poultry $(26 \%)$, bovine $(25 \%)$, caprine $(8 \%)$, porcine $(6 \%)$, ovine $(2 \%)$, and camelid $(0.5 \%)$. Some animals were exposed to $>1$ pesticide product and some pesticide products had $>1$ ingredient class. The pesticide category with the highest number of exposed animals was insecticides $(2,151)$, followed by herbicides (839), rodenticides (765) and fungicides (286). The treemap below illustrates the number and proportions of animals exposed to the 4 pesticide categories and the top 3 pesticide classes within each category based on the first active ingredient. For all pesticide exposures in all species, no illness was reported in $68 \%$ of animals. According to assessment by APCC staff, only $35 \%$ (333) of animals showing clinical signs were considered with confidence (medium or high likelihood) to be due to pesticide exposure. For these 333 animals, severity of illness was mild for $80 \%$ (266 animals), moderate for 18\% (61 animals), major for $1 \%$ (3 animals) and caused death in $1 \%$ (3 animals). Among animals with confidence that clinical signs were due to pesticide exposure the most frequent syndrome was dermatologic.

\section{Conclusions}

Suspected pesticide exposure was the most frequent reason a call concerning livestock was made to the APCC. Callers reported that most animals showed no illness, and major illness or death was rare. Livestock were most frequently exposed to the insecticide category, and $46 \%$ of the animals with exposure to insecticides were exposed to the pyrethrin class. This is consistent with the phasing out of organophosphate insecticides for residential use since 2000 and the increasing use of pyrethrin insecticides ${ }^{3}$, which are considered less toxic. Limitations of this study include: 1) data from only one major animal poison control hotline was available for analysis and people may call their veterinarian directly or use the internet 2) calls regarding specific ingredients may be over represented due to corporate client relationships with the APCC 3) illness may have occurred after the call was made, therefore the proportion of animals with illness following suspected exposure may be an underestimate.

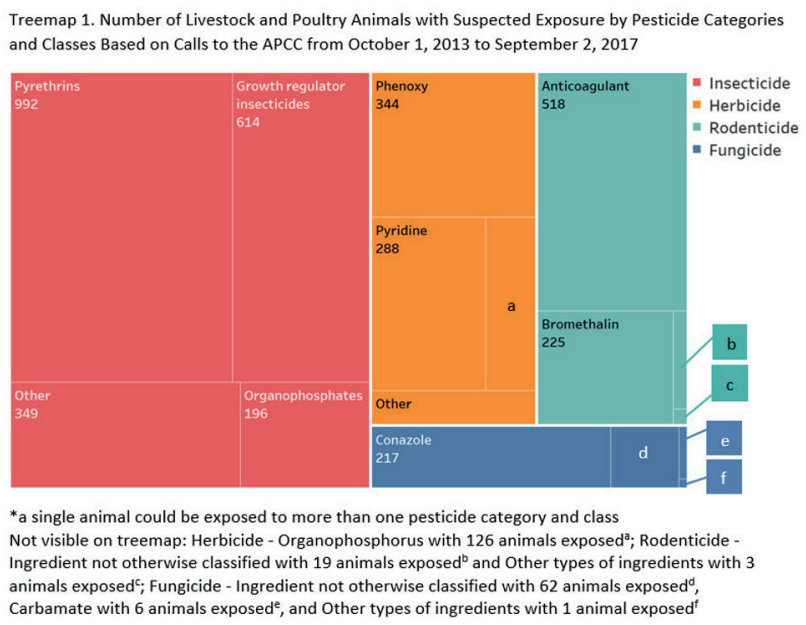

\section{Keywords}

pesticide poisoning; livestock; poison control center; animal health

\section{Acknowledgments}

Copyright $(2017$ The American Society for the Prevention of Cruelty to Animals (ASPCA).

All Rights Reserved. 
References

1. Wang Y, Kruzik P, Helsber A, Helsberg I, Rausch W. (2006) Pesticide poisoning in domestic animals and livestock in Austria: A 6 year retrospective study. Forensic Science International 169:157-160.

2. Gwaltney-Brant SM. (2012). Epidemiology of Animal Poisonings in the United States. In: Gupta RC (Ed.), Veterinary Toxicology: Basic and Clinical Principles. Elsevier, Second ed: 80-87.

3. Power LE, Sudakin DL. Pyrethrin and pyrethroid exposures in the United States: A longitudinal analysis of incidents reported to Poison Centers. (2007) J of Medical Toxicology. 3(3):94-99.

\section{*Judy Akkina}

E-mail: judy.e.akkina@aphis.usda.gov 